\title{
Estudio al sector calzado de Tungurahua en el Marco de la ISO 26000
}

\author{
Erika Tapia -Panchi' ${ }^{1}$, Carmen Tisalema- Bravo ${ }^{2}$, Pamela Miranda- Lozada ${ }^{3}$
}

Fecha de recepción: 2 de Febrero 2018

\section{Resumen}

Este trabajo evalúa el nivel de la Responsabilidad Social en las empresas del sector calzado desde la perspectiva de sus trabajadores y sus consumidores, se muestran también los beneficios y barreras a los que las empresas se enfrentan, y las dimensiones de Responsabilidad Social que están enmarcadas dentro de la Norma ISO 26000. Los resultados mostraron la relación entre las dimensiones: económica, legal, ética, ambiental y social con un coeficiente de path superior al $50 \%$ respecto a los trabajadores, mientras que para la perspectiva de los consumidores existe una relación superior al 54\% excepto entre las dimensiones ética y ambiental.

Palabras clave: Responsabilidad social, Norma ISO 26000, trabajadores, consumidores.

\section{Abstract}

This work evaluates the level of Social Responsibility in companies in the footwear sector from the perspective of its workers and consumers; it also shows the benefits and barriers that companies face, and the dimensions of Social Responsibility that are framed within of the ISO 26000 Standard. The results showed the relationship between the dimensions: economic, legal, ethical, environmental and social with a path coefficient superior to $50 \%$ with respect to workers, while for the consumers' perspective there is a relationship superior to $54 \%$ except between the ethical and environmental dimensions.

Keywords: Social responsibility, ISO 26000 Standard, workers, consumers

\section{Introducción}

La Responsabilidad Social es un tema que ha venido tomando protagonismo en los últimos años en la actividad empresarial y comercial. Aquellas empresas que orienten sus actuaciones de RSE hacia sus trabajadores y consumidores conseguirán un doble efecto. Por una parte, lograrán mejorar la actitud y el entusiasmo del trabajador hacia la empresa. Y en el consumidor incrementar la intención de compra futura de sus productos frente a otros que provengan de empresas que no atiendan suficientemente estas responsabilidades.

Las empresas donde se aplican prácticas de la Responsabilidad Social poseen ventajas como: el reconocimiento en el mercado, mayor competitividad e incremento en sus ingresos, ya que implementan en sus políticas objetivos y compromisos hacia la mejora entre sus trabajadores. Protegiendo su calidad de vida personal, profesional y ético social. Los objetivos están relacionados con la equidad, igualdad, respeto, salarios justos y cumplimiento de los derechos y obligaciones de los trabajadores cubriendo las expectativas de la empresa. (Sotelo \& Garcia, 2014)

La implementación de la responsabilidad social por parte de las empresas de calzado beneficia en su imagen frente al consumidor que se ven reflejadas en la lealtad de los mismos, los factores que explican la lealtad son la imagen del establecimiento, la convivencia de la relación comercial y la búsqueda de ahorro por parte de los consumidores. (Paiva, Sandoval \& Michele, 2012)

\section{Metodología}

En el presente estudio se utilizó la metodología cuantitativa, en la perspectiva de los trabajadores se utilizó una población de 15 empresas del sector calzado registradas en la Superintendencia de Compañías de las cuales se tomó de 3 a 5 trabajadores de cada empresa para la
Fecha de aceptación: 28 de Marzo 2018

aplicación de las encuestas, debido a que su aplicación fue en el mes de noviembre donde las empresas están en temporada alta y se dio la apertura de solo 3 trabajadores de cada una de las empresas logrando obtener un total de 65 encuestados, mientras que para la perspectiva de los trabajadores, y para los consumidores se aplicó a un total de 384 encuestas a consumidores del sector calzado de Tungurahua de los cantones de Ambato y Cevallos, al ser los cantones donde más producción de calzado existe, y se encuentran registrados en la Superintendencia de Compañías.

Debido a que la población de estudio es extensa, será escogida de manera aleatoria se calculará una muestra con una población finita $\mathrm{n}$, con un valor de confianza del 95\% y un margen de error del $5 \%$

Dónde:

$\mathrm{n}=504.583$

$Z=1,96$

$\mathrm{P}=0,05$

$\mathrm{e}=0,05$

$\mathrm{i}=0,05$

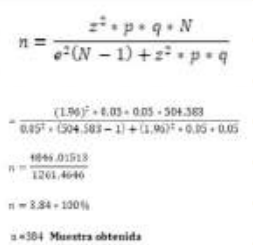

A través de tablas de frecuencia y gráficos con descripción estadística se mostrarán los resultados obtenidos de acuerdo a las encuestas aplicadas. Para la comprobación de la hipótesis se aplicó el coeficiente de path.

\section{Resultados}

Dimensiones de la Responsabilidad Social

De acuerdo con Aguilera \& Puerto (2012), en su investigacion mencionan las siguientes dimensiones de la Responsabilidad Social:

- Económico: Constituye en la producción de bienes y servicios que satisfacen las necesidades de la sociedad.

- Legal: Implica el cumplimiento de la leyes locales, nacionales e internacionales que den credibilidad a los inversionistas, proveedores y consumidores.

- Ética: El cumplimiento de los lineamientos relacionados con la conducta y valores de las organizaciones

- Social: Donaciones, auspicios de dinero y otros recursos para sectores vulnerables de la sociedad

- Ambiental: Protección y cuidado de los seres vivos existentes en el mundo.

\section{NORMA INTERNACIONAL ISO 26000}

La Norma ISO 26000 tiene como objetivo dar una orientación en temas de responsabilidad social a las organizaciones y empresas para que contribuyan al desarrollo sustentable y sostenible y la inclusión de grupos vulnerables a través de la ética. (ISO 26000, 2010).

Con la aplicación de las Normas ISO 26000 las empresas lograrían un equilibrio satisfactorio que favorezca en este caso a sus trabajadores a través de la aplicación de prácticas que enmarquen las materias fundamentales donde establecen: Derechos Humanos y Prácticas Laborales justas, Asunto de consumidores y Participación activa y desarrollo de la comunidad que se relacionan con los beneficios de los trabajadores y a sus consumidores (Cea Moure, 2013).

\section{Respeto de los intereses de los trabajadores}

El respeto a los intereses de los trabajadores es un término que ayuda a asegurar que las personas sean tratadas con dignidad y respeto en

1'Ingeniera en Finanzas y Auditoría CPA. Docente de la Facultad de Contabilidad y Auditoría de la Universidad Técnica de Ambato. Ambato - Ecuador. E- Mail: ep.tapia@uta.edu.ec ${ }^{2}$ Estudiante de la Carrera de Economía de la Facultad de Contabilidad y Auditoría de la Universidad Técnica de Ambato. Ambato - Ecuador. E- Mail: tisalemacarmen@hotmail.com ${ }^{3}$ Estudiante de la Carrera de Economía de la Facultad de Contabilidad y Auditoría de la Universidad Técnica de Ambato. Ambato - Ecuador. E- Mail: pameslozada@gmail.com 
el lugar de trabajo. También refuerza el compromiso de cumplir con las leyes que prohíben la discriminación, el acoso y la represalia. Los empleadores deben identificar y corregir los problemas de seguridad y salud, lo que exige que los empleadores eliminen o reduzcan los peligros primero introduciendo cambios factibles en las condiciones de trabajo. (Aguilar, 2014)

\section{Respeto de los intereses de los consumidores}

La idea de que el mercado sirve a los derechos y a las necesidades de los seres humanos y que sus intereses se ubican en el centro mismo de la actividad comercial. Siendo que los intereses que tengan los consumidores en relación con las empresas son el eje para que estos efectúen su compra (Rodríguez, 2014).

A continuación se muestran los resultados obtenidos:

\section{Trabajadores}

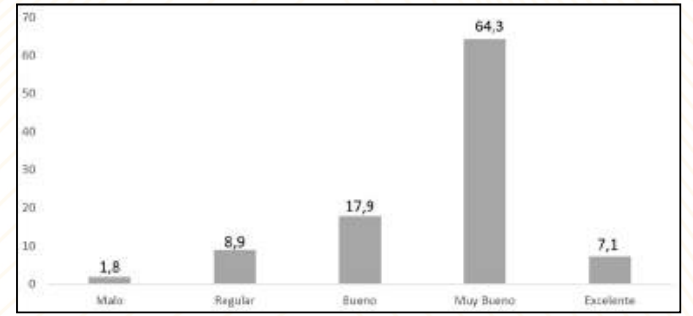

Fuente: Elaboración propia a partir de la encuesta a trabajadores del sector calzado de la provincia de Tungurahua.

Figura 1. Grado de Involucramiento de los trabajadores

El grado de involucramiento que tiene la empresa con sus trabajadores muestran los siguientes resultados: de un $64,3 \%$ con un calificativo de muy bueno, un $17,9 \%$ lo califican como bueno y un porcentaje del $7,1 \%$ como excelente, lo que indica que las empresas están cumpliendo con un porcentaje aceptable con aplicación de las normas de responsabilidad social empresarial enmarcadas en la ISO 26000 en su apartado de involucramiento de las partes interesadas en este caso los trabajadores.

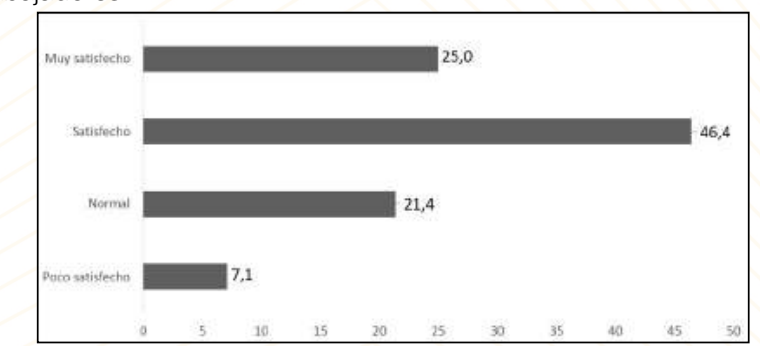

Fuente: Elaboración propia a partir de la encuesta a trabajadores del sector calzado de la provincia de Tungurahua. Figura 2. Clima laboral

En lo referente al clima laboral el $46,4 \%$ de los trabajadores encuestados se siente satisfecho del clima laboral en la empresa, el 25\% están muy satisfecho, lo que indica que el respeto en el trabajo de igual a igual es beneficioso para mantener un clima laboral agradable dando cumplimiento a la norma ISO 26000 de diálogo social y por ende el respeto a los trabajadores de esta manera evitando violencia.

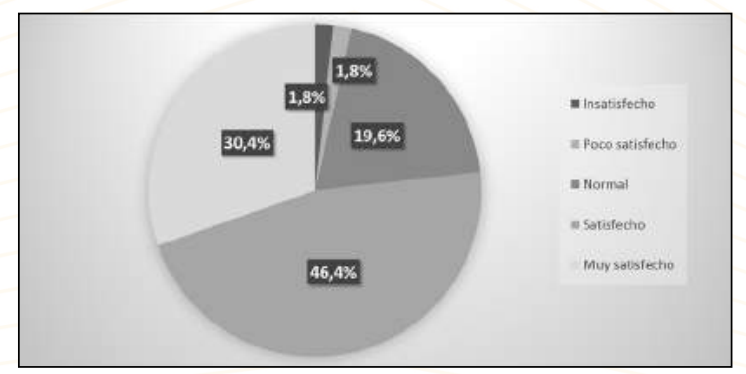

Fuente: Elaboración propia a partir de la encuesta a trabajadores del sector calzado de la provincia de Tungurahua.

Figura 3. Reglamentos de seguridad ocupacional
En cuanto a los reglamentos de seguridad ocupacional el 46,4\% están satisfechos, el 30,4\% muy satisfechos y el 19,6\% normal, es decir las normas y estatutos de la empresa cubren las necesidades de seguridad de los trabajadores con lo cual las empresas cumplen con las leyes nacionales y de trabajo y además con las Normas ISO 26000 que indican que los empleadores deben proporcionar salud y seguridad social a sus empleados.

\section{Consumidores}

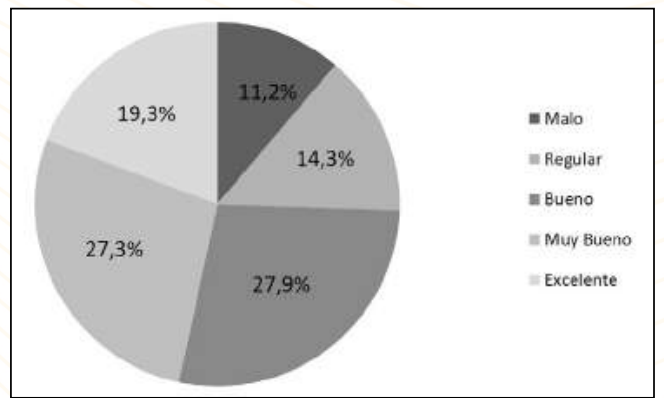

Fuente: Elaboración propia a partir de la encuesta a consumidores del sector calzado de la provincia de Tungurahua

Figura 4. Canales de comunicación empresa-consumidor

En cuanto a los canales de comunicación empresa-consumidor se aprecia que del total de los encuestados con respecto, a si la empresa tiene canales de comunicación empresa-consumidor en caso de sugerencias y reclamos se obtuvo que 107 que corresponde al 27,9\% respondieron que es bueno, 105 que representa el $27,3 \%$ respondieron que es muy bueno, 74 que representa el 19,3\% respondieron que es excelente. Es así que se evidencia que existe cumplimiento del apartado de la NORMA ISO 26000, que es el 6.7.6 Servicios de atención al cliente, apoyo y resolución de quejas y controversias.

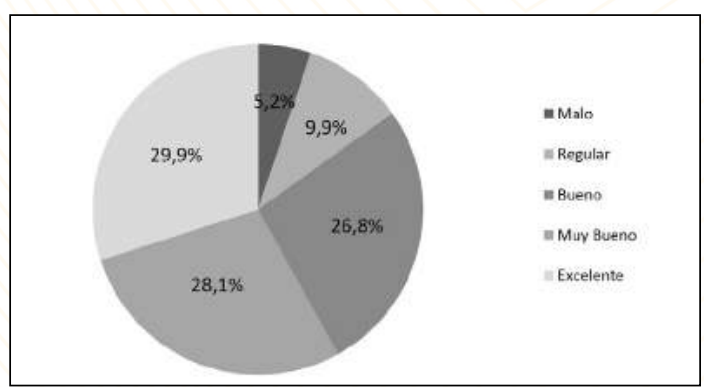

Fuente: Elaboración propia a partir de la encuesta a consumidores del sector calzado de la provincia de Tungurahua Figura 5. Prácticas justas de Marketing

Con respecto a las prácticas justas de marketing de las empresas del sector calzado 115 que representa el 29,9\% respondieron que son excelentes, 108 que representa el 28,1 que son muy buenas, 103 que representa el $26,8 \%$ que son buenas. Los resultados obtenidos muestran que se está cumpliendo con el apartado de la ISO 26000 6.7.3 Prácticas justas de marketing, información objetiva e imparcial.

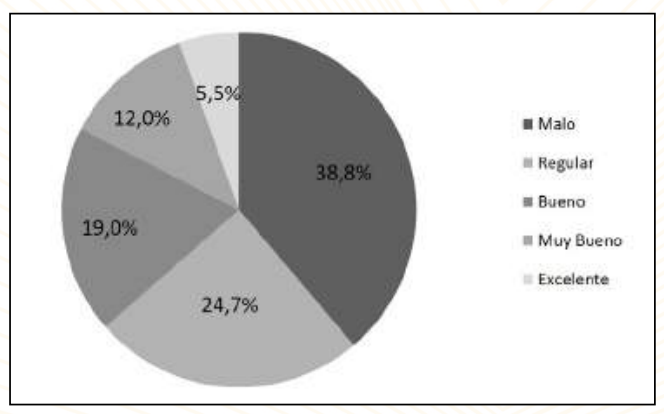

Fuente: Elaboración propia a partir de la encuesta a consumidores del sector calzado de la provincia de Tungurahua Figura 6. Apoyo en programas solidarios 
Con respecto a si las empresas de calzado brindan apoyo en programas solidarios y a fundaciones 149 que representa el 38,8\% respondieron que es malo, 95 que representa el $24,7 \%$ que es regular, 73 que representa el $19 \%$ que es bueno. Los resultados obtenidos reflejan la relación con el apartado de la ISO 26000, 6.8.3 Participación activa de la comunidad, el cual refleja que no existe cumplimiento por parte de las empresas de calzado.

\section{Comprobación de hipótesis trabajadores}

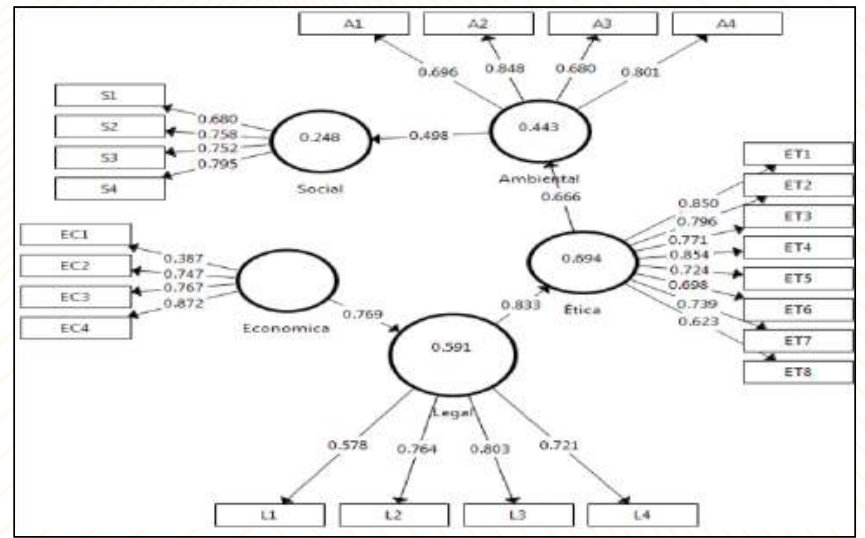

Fuente: Elaboración propia a partir de la encuesta a trabajadores del sector calzado de la provincia de Tungurahua.

Figura 7. Medición de las dimensiones de Responsabilidad Social de los trabajadores

H1: La situación económica de las empresas de calzado influye en un adecuado cumplimiento de disposiciones legales. Al obtener una correlación de 0.769 (76,9\%) podemos manifestar que la hipótesis esta verificada con un alto nivel de aceptación.

H2: La empresa de calzado que cumple con las disposiciones legales tendrá un adecuado comportamiento ético en la sociedad, el porcentaje alcanzado es de 0.833 (83,3\%) dando lugar a que la hipótesis planteada se acepte ya que supera el valor mínimo de coeficiente de Path.

H3: Al tener un comportamiento ético las empresas del sector calzado tienen una influencia positiva en el desarrollo de actividades de protección ambiental. Al tener un valor entre la dimensión ética y ambiental de $0.666(66,6 \%)$ podemos plantear que la hipótesis planteada se acepta.

H4: Las acciones que desarrollan las empresas del sector calzado en protección del medio ambiente favorecen al desarrollo social y mejoran de la calidad de vida de las comunidades aledañas donde realizan sus actividades. Al obtener un valor de 0.498 (49,8\%) se puede demostrar que la hipótesis establecida tiene alto nivel de aceptación ya que supera el límite establecido en el coeficiente de Path que es 0.30 (30\%).

\section{Comprobación de hipótesis consumidores}

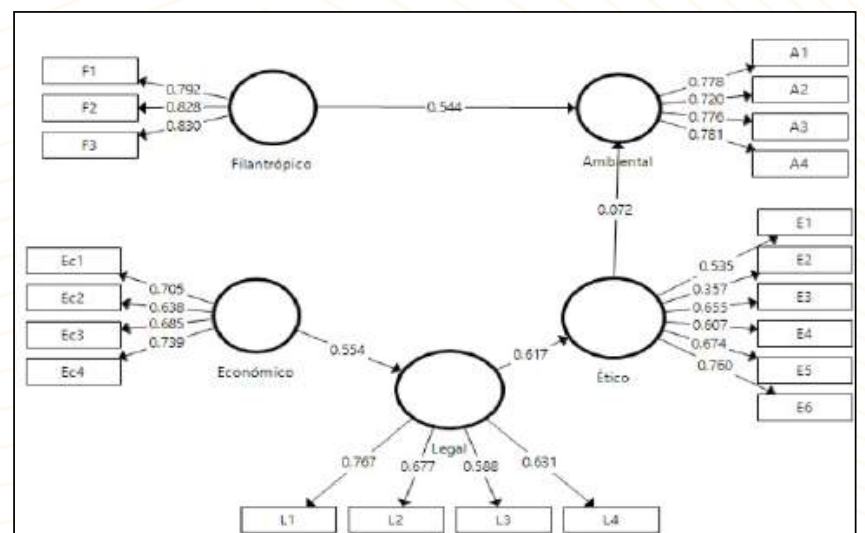

Fuente: Elaboración propia a partir de la encuesta a consumidores del sector calzado de la provincia de Tungurahua.
H1: De acuerdo al manejo de la situación económica que muestran las empresas de calzado se demuestra que existe coherencia con el cumplimiento de la parte legal. Los resultados muestran que existe un $55 \%$ $(0,554)$ de correlación entre las dimensiones económica y legal por lo que se refleja que la hipótesis es aceptada.

H2: En este sentido se ha podido comprobar que las empresas de calzado cumplen con las disposiciones legales en cuanto a sus actividades lo cual pone en manifiesto su correcto comportamiento ético con la sociedad. Los resultados revelan un peso estadísticamente significativo del $62 \%(0,617)$ lo cual es capaz de explicar que la hipótesis planteada es aceptada.

H3: La respuesta de los consumidores de acuerdo al cumplimiento ético relacionado al respeto del medio ambiente no muestra consistencia positiva de criterios. Los resultados demuestran que la dimensión ética no tiene relación con la dimensión ambiental, el valor obtenido es de $7,2 \%(0,072)$ podemos demostrar que la hipótesis planteada se rechaza ya que no cumple los parámetros de aceptación.

H4: En cuanto al respeto que muestran las empresas del sector calzado con el medio ambiente en el desarrollo de sus actividades cabe reconocer que contribuyen para que los consumidores perciban el buen papel que están haciendo dichas empresas y así fortalecen sus relaciones comerciales. Los resultados evidencian la relación positiva existente entre la dimensión ambiental y filantrópica con un 54\% $(0,544)$ con lo cual verificamos que la hipótesis es aceptada.

\section{Conclusiones}

Las dimensiones de Responsabilidad Social que el sector de calzado de Tungurahua practica con sus trabajadores son: Las dimensiones Económica, Legal, Ética, Ambiental y Social, la práctica de actividades que enmarcan estas dimensiones hacen que las empresas de estudio sean competitivas en el mercado con el resto de empresas.

Las empresas que aplican actividades socialmente responsables gozan de beneficios como: un clima laboral agradable ente sus trabajadores lo que hace que aumente la producción minimizando el tiempo, mermar la discriminación logrando igualdad de oportunidades para todos y estabilidad para los trabajadores, condiciones de trabajo según los reglamentos de seguridad, la inclusión de los trabajadores en aspectos de conciliación familiar laboral y social de los trabajadores por otro lado la relación de valoraciones de la satisfacción al consumidor, precios de los productos acordes a la calidad, posicionamiento en mercado (marca reconocida), investigación de nuevas demandas de los consumidores, respeto y atención a los clientes, privacidad de la información personal, presentar publicidad responsable no engañosa, dar a conocer la información transparente de sus actividades, cumplir con la emisión de facturas, son las que hacen que estas sean preferidas por los consumidores Sin embargo existen empresas que tiene que eliminar barreras para la aplicación de prácticas de responsabilidad social entre las más comunes son temas ambientales y el valor económico de la empresa con la sociedad.

Finalmente, se concluye que el nivel de las empresas del sector calzado de Tungurahua en temas de Responsabilidad Social en el marco de la ISO 26000 están en un promedio aceptable, en vista que las dimensiones legal-ética es donde las empresas han tenido más relevancia con un coeficiente de Path del $83,3 \%$ seguido por la dimensión económica- legal con el $76,9 \%$, y en lo que se refiere a la dimensión ambiental y social con un porcentaje menor a las dimensiones anteriores pero aceptable del $49,80 \%$ desde la perspectiva de los trabajadores. Además se evidenció la relación entre las dimensiones económico-legal 55\%, filantrópico-ambiental 54\%, legal-ético $62 \%$ desde la perspectiva de los consumidores, lo que indica que las empresas del sector calzado velan los beneficios personales, económicos y legales de los trabajadores y consumidores. 


\section{Referencias}

Aguilera , A., \& Puerto , D. P. (Marzo de 2012). Crecimiento empresarial basado en la Responsabilidad Social. Pensamiento \& Gestión, 4.

Cea Moure, R. (14 de Noviembre de 2013). repositorio.uam.es. Obtenido de repositorio.uam.es: https://repositorio.uam.es/bitstream/handle/10486/4183/28409_cea_moure_ramiro.pdf?sequence=1

ISO 26000. (2010). Guia de responsabilidad social. Suiza: Aenor.

Paiva, G., Sandoval, M., \& Michele, B. (2012). Factores explicativos de la lealtad de clientes de Iso supermercados. INNOVAR, 153-164.

Rodríguez, J. (2014). De los intereses d elos consumidores y de la protección de la libre competencia en los mercados: Un estudio sobre las modalidades de competencia desleal en Colombia y sis medidas de protección. Cali: Universidad Catolica de Colombia.

Sotelo, J., \& Garcia, J. (2014). La responsabilidad social en empresas del sector cuero-calzado y su disposición a compartir los beneficios con los colaboradores. Ciencias Administrativas. Teoría y Praxis, 16.

\section{Anexos página 26}

